\title{
346
}

\section{Stiffperson syndrome and type 1 diabetes mellitus}

Antonio F Oliveira Filho (1) Rossana C Almeida (1) Eveline M C Brito (2) Adriana B Nunes (3)

(1) Health Service Pombal, Pombal, PB;(2) Federal University Pernambuco, Recife, PE;(3) Federal University Rio Grande Norte, Natal, RN, Brazil

Stiff person syndrome (SPS) is a rare disease characterized by progressive muscle rigidity and spasms, which can lead to functional disability. This condition can be challenging to diagnose if there is no high index of suspicion. Its etiology is unknown, but one of the most likely causes is autoimmune aggression, which is corroborated by that fact that it is associated with other autoimmune diseases in half of the cases described in literature

\section{OBJECTIVES}

We will present here the case of a boy in which SPS is associated to type I diabetes mellitus.

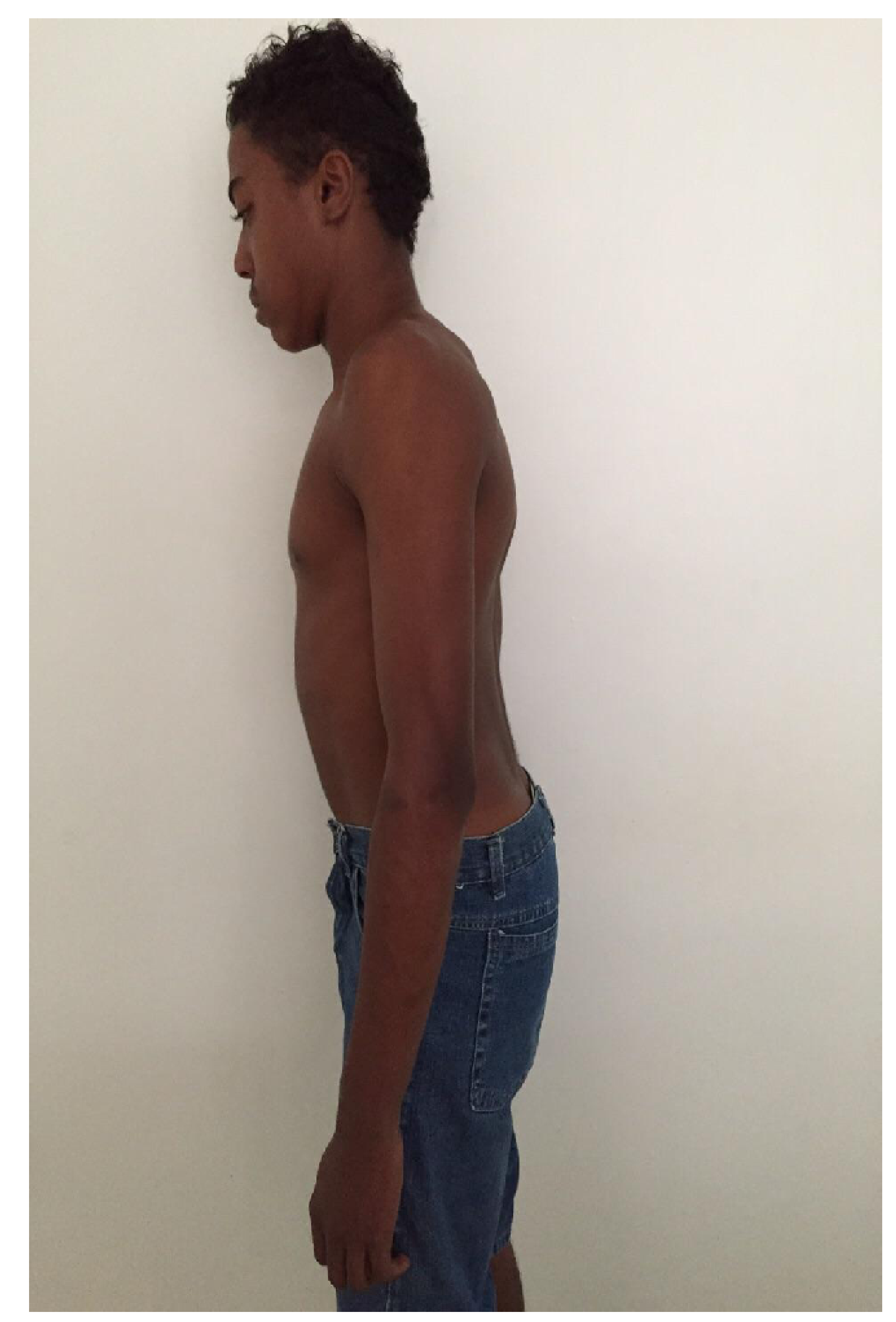

\section{RESULTS / CLINICAL REPORT}

The 15-year-old patient was diagnosed with type I diabetes 5 years ago, when he presented with altered level of consciousness and capillary glucose of $32.22 \mathrm{mmol} / \mathrm{L}$. Three years ago he started presenting with episodes of muscle stiffness and spasms, triggered by touch and lying down position, which led him to sleep sitting on a chair. After thorough investigation, including a electroneuromyography exam showing continuous motor stimulation and immunological testes indicating high levels of anti-GAD antibodies $(50 \mathrm{IU} / \mathrm{ml})$, SPS became the main diagnostic hypothesis.

The patient has been currently using Clonazepam, with overall improvement of the symptoms. He also uses NPH and regular insulin for the diabetes, and does not suffer from any chronic complications.

\section{COMMENTARY}

SPS often occurred in conjunction with a variety of autoimmune diseases. These include thyroiditis, vitiligo, pernicious anemia, and, particularly, type 1 diabetes. Otherwise, autoimmune mechanism was provided by the observation of polyclonal and oligoclonal IgG antibody elevations in the cerebrospinal fluid of the majority of patients with SPS frequently targeting GABAergic (gamma amino butyric acid) neurons and their nerve terminals. The presence of type I diabetes on this patient, who already suffered from a neuromuscular disease of unknown etiology, brought to light the hypothesis of SPS, later confirmed by laboratory exams. This allowed appropriate treatment in time. Therefore, this case illustrates the importance of having a broader view in clinical practice, taking co morbidities into consideration in order to reach the correct diagnosis. 